\title{
Some methods to improve the utility of conditioned Latin Hypercube sampling
}

\author{
Brendan P Malone Corresp., $^{1,2}{ }^{2}$, Budiman Minansy ${ }^{2}$, Colby Brungard ${ }^{3}$ \\ ${ }^{1}$ CSIRO, Agriculture and Food, Canberra ACT, Australia \\ 2 The Sydney Institute of Agriculture, The University of Sydney, Sydney, New South Wales, Australia \\ 3 Plant and Environmental Sciences, New Mexico State University, Las Cruces, New Mexico, United States \\ Corresponding Author: Brendan P Malone \\ Email address: brendan.malone@sydney.edu.au
}

The conditioned Latin hypercube sampling (cLHS) algorithm is popularly used for planning field sampling surveys in order to understand the spatial behavior of natural phenomena such as soils. This technical note collates, summarises, and extends existing solutions to problems that field scientists face when using cLHS. These problems include optimizing the sample size, re-locating sites when an original site is deemed inaccessible, and how to account for existing sample data, so that under-sampled areas can be prioritized for sampling. These solutions, which we also share as individual R scripts, will facilitate much wider application of what has been a very useful sampling algorithm for scientific investigation of soil spatial variation. 
1 Some methods to improve the utility of conditioned Latin Hypercube sampling

2

3 Brendan P. Malone*

4 CSIRO Agriculture and Food

5 Sydney Institute of Agriculture, The University of Sydney

6 brendan.malone@csiro.au

7

8 Budiman Minasny

9 Sydney Institute of Agriculture, The University of Sydney

10 budiman.minasny@sydney.edu.au

11

12 Colby Brungard

13 Plant and Environmental Sciences, New Mexico State University.

14 cbrung@ad.nmsu.edu

15

*Corresponding author

17

18

19

20

21

22

23

24

25

26 


\section{Abstract}

The conditioned Latin hypercube sampling (cLHS) algorithm is popularly used for planning field sampling surveys in order to understand the spatial behavior of natural phenomena such as soils. This technical note collates, summarises, and extends existing solutions to problems that field scientists face when using cLHS. These problems include optimizing the sample size, re-locating sites when an original site is deemed inaccessible, and how to account for existing sample data, so that under-sampled areas can be prioritized for sampling. These solutions, which we also share as individual R scripts, will facilitate much wider application of what has been a very useful sampling algorithm for scientific investigation of soil spatial variation.

\section{Introduction}

The conditioned Latin hypercube sampling (cLHS) algorithm (Minansy and McBratney 2006) was designed with digital soil mapping (DSM) in mind. cLHS is a random stratified procedure that choses sampling locations based on prior information pertaining to a suite of environmental variables in an givenarea. cLHS has been used extensively in digital soil mapping projects throughout the world with recent examples in the last 5 years including Sun et al. (2017) in China, Jeong et al. (2017) in Korea, Scarpone et al. (2016) in Canada, and Thomas et al. (2015) in Australia. cLHS has also been used for other purposes and contexts too. For example in optimal soil spectral model calibration (Ramirez-Lopez et al. 2014, Kopačková et al. 2017), understanding the conditions which determine Phytophthora distribution in rainforests (Scarlett et al. 2015), and assessing the uncertainty of digital elevation models derived from Light detection and ranging (LiDAR) technology (Chu et al. 2014).

For DSM, the algorithm exploits collections of environmental variables pertaining to soil forming factors and proxies thereof (McBratney et al. 2003; e.g. digital elevation model derivatives, remote sensing imagery of vegetation type and distribution, climatic data, and geological maps) to derive a sample configuration (of specified size), such that the empirical distribution function of each environmental variable is replicated (Clifford et al. 2014). 
55 Presuming that soil variation is a function of the chosen environmental variables, it is reasoned 56 that models fitted using data collected via cLHS, capture all the soil spatial variability and will 57 be applicable across the whole spatial extent to be mapped.

58

However, our own experience, and from personal communication with other researchers and field technicians, a common set of methodological questions that arise when using cLHS. These questions are:

1. How many samples should I collect?

2. Where else can I sample when a cLHS location cannot be visited because of difficult terrain, locked gate, safety reasons etc.?

3. How do I account for existing samples when designing a new survey?

The purpose of this technical note is to describe solutions to each of these questions. We first begin with a brief overview of the cLHS algorithm and then address each question separately.

\section{Materials and Methods}

\section{A short overview of cLHS}

Conditioned Latin Hypercube sampling is one of the many environmental surveying tools available for understanding the spatial characteristics of environmental phenomena. Extended discussions about soil sampling, surveying and monitoring of natural resources in a broad context can be found in seminal publications such as de Gruijter et al (2006) and Webster and Lark (2012). Conditioned Latin Hypercube sampling has its origins in Latin Hypercube sampling (LHS) first proposed by McKay et al. (1979). LHS is an efficient way to reproduce an empirical distribution function, where the idea is to divide the empirical distribution function of a variable, $X$, into $n$ equi-probable, non-overlapping strata, and then draw one random value from each stratum. In a multi-dimensional setting, for $k$ variables, $X_{1}, X_{2}, \ldots, X_{k}$, the $n$ random values drawn for variable $X_{1}$ are combined randomly (or in some order to maintain its correlation) with the $n$ random values drawn for variable $X_{2}$, and so on until $n k$-tuples are formed, i.e., the Latin hypercube sample (Clifford et al. 2014). Its utility for soil sampling was noted by Minasny and McBratney (2006), but they recognised that some generalisation of LHS sampling was required so that selected samples actually existed in the real world. Subsequently, they proposed a 
83 conditioning of the LHS, which is achieved by drawing an initial Latin hypercube sample from 84 the ancillary information, then using simulated annealing to permute the sample in such a way that an objective function is minimised. The objective function of Minasny and McBratney (2006) comprised three criteria:

1. Matching the sample with the empirical distribution functions of the continuous ancillary variables

2. Matching the sample with the empirical distribution functions of the categorical ancillary variables; and

3. Matching the sample with the correlation matrix of the continuous ancillary variables.

See Minasny and McBratney (2006) for full detailing of the cLHS algorithm.

\section{How many samples should I collect?}

Ironically this question arose from a situation where we examined data from a $\sim 100 \mathrm{ha}$ field. Within this field 238 soil samples were collected by regular grid sampling. Soil samples were then analysed for key soil properties. Also collected from the field were ancillary data including soil conductivity (from an EM38 sensor), elevation, and crop yield data. Our interest was in determining whether the 238 soil samples adequately covered the ancillary data space and if an alternative sampling configuration using cLHS could be used to determine a specific sample number that would achieve the same data coverage as the 238 sites.

We acknowledge that for most contexts, practitioners will not experience the same situation we have just described, but will only have a suite of ancillary data and will need to estimate an optimal sample number.

The solution to choosing an optimal number of samples is to compare the empirical distribution functions of incrementally larger sample sizes with those of the population. The resulting output will display a typical exponential growth (or decay) curve, depending on what comparative metric is used, that plateaus at some point with increasing sample size. This allows one to invoke a diminishing returns-like rule to derive an optimal sample number. This approach was exemplified in both Ramirez-Lopez et al. (2014) for selection of a number of observations to 
111 optimise the fitting of soil spectral models, and Stumpf et al. (2016) for evaluating an optimal

112 sample size to calibrate a DSM model.

113 Comparing the empirical distribution functions of the samples and the population can be done in 114 a number of ways. Stumpf et al. (2016) compared the overall variance of the ancillary data from 115 an entire region, hereinafter referred to as the population, with that of the sample. The 116 application of this approach for categorical data was not established, but for categorical data it is 117 only necessary to match the relative proportions of the categories. Other metrics for continuous 118 variables could involve comparison of the quantiles of the empirical distributions. This would 119 require comparing an absolute deviation between the quantiles (which could be absolute 120 difference or Euclidean distance) for each ancillary variable, then deriving a unified value by 121 combining the distances (weighted averaging) from each variable. Alternatively, comparison 122 could be made of the principal components (PC) of the ancillary variables (using the approach 123 described by Krzanowski (1979)), which would alleviate the need to first compute distances for 124 each variable and then combine into a single measure of distance.

125 Probably a more standard approach for comparing empirical distribution functions is the 126 Kullback and Leibler (1951) divergence which is specifically designed for comparing 127 distributions (Clifford et al., 2014). The Kullback-Leibler (KL) divergence (also called relative 128 entropy) compares the relative proportions of samples within each histogram bin of the 129 distributions. The KL divergence can be computed as:

where $E_{i}$ is the distribution of an ancillary variable for the population ( $i$ is a histogram bin), and $O_{i}$ is the distribution of the same ancillary variable from a sample.

135 The KL divergence decreases towards zero as the population and sample distributions converge 136 (Clifford et al. 2014) and can be defined for both categorical and continuous ancillary data.

137 Signal processing is probably the most common use case for KL divergence, such as the 138 characterisation of relative entropy in information systems where the related Shannon's 
139 information criterion (Shannon and Weaver, 1949) is widely applied. Although useful, the KL

140 divergence is not incorporated into the Minasny and McBratney (2006) cLHS algorithm, where

141 instead comparison is made using the quantity: $\sum_{i}\left|O_{i}-E_{i}\right|$. This metric could also be useful for

142 optimisation of a sample size too, but the difference between it and the KL divergence is that it

143 penalises all departures from the population values equally. With the KL divergence, the penalty

144 of missing a sample from the tail of a distribution is larger than the penalty of missing a sample

145 close to the mode.

146 In the following, we demonstrate the usage of KL divergence to help identify an optimal sample

147 size in the $\sim 100$ ha field previously described. The cLHS algorithm was run sequentially with

148 increasing sample sizes beginning at 10 and finishing at 500. A step size of 10 samples was used.

149 Each sample size was repeated 10 times to assess the dispersion about the mean KL divergence

150 estimate. KL divergence was estimated using a bin number of 25 for each ancillary variable and

151 then aggregated for all variables by calculating the mean. Note that the justification for selecting

152 a bin number of 25 was to replicate the settings as implemented Clifford et al. (2014). Our own

153 small investigations indicated this bin number to be adequate for our data, but would recommend

154 for other studies and contexts to evaluate the KL divergence response to various bin numbers via

155 iteration. Too few bins may not adequately capture the main features of a distribution, giving

156 misleading low divergence values. While too many bins maybe overly sensitive to noise and

157 provide high KL divergence values in otherwise near matching distributions.

158 In this work, The R package clhs (Roudier 2011) was used for performing the cLHS. The inputs

159 into the $c l h s$ function included a table form ( $\mathrm{R}$ data.frame) of the ancillary data that was collected

160 in the field of interest, i.e. the proximal sensed data and yield data. We used the default 10000

161 iterations of the annealing process to run the function. A schematic of the sample size

162 optimisation algorithm is given in Figure 1.

164 [Insert Figure 1 around here]

165 Figure 1. Schematic of sample number size optimisation algorithm. 
167 168

169

170

171

172

173

174

175

176

177

178

179

180

181

182

183

184

185

186

187

188

189

190

191

192

193

194

195

Where else can I sample when a cLHS location cannot be visited because of difficult terrain, locked gate, safety reasons, etc.?

One of the biggest criticisms levelled at cLHS is the rigidness around the sampling configuration. For example both Thomas et al. (2012) and Kidd et al. (2015) remarked on the lack of guidance on what to do when a site cannot be accessed. They arrived at the same conclusion, that rather than continuing with cLHC sampling, the more flexible sampling approach of fuzzy stratified sampling be used. Kidd et al. (2015) demonstrated that this alternative sampling approach was comparable to cLHS in their work. Nevertheless, once a sampling location selected by the cLHC algorithm is unable to be visited for any number of reasons, a replacement site needs to be selected. The introduction of replacement sites can potentially degrade the objective of cLHS if the replacement sites are arbitrarily selected in nearby locations that are accessible.

Modification of the cLHS objective function can be done so that accessibility is considered. This has been done by Roudier et al. (2012) and Mulder et al. (2013) where the cost of getting to sampling locations was incorporated into the annealing schedule of the cLHS algorithm. Such modifications however, do not guarantee accessibility but do increase the probability that a site will be accessible. Adhering to an original cLHS site configuration, Stumpf et al. (2016) selected alternative sample sites by deriving numerous test sample sets based on the original cLHS sites with all possible combinations of the covariate data from the cLHS strata. They selected the test sample set that most closely represented the original cLHS sites via quantile matching. Implementing this method would require prior knowledge of accessible and inaccessible sites, which for Stumpf et al. (2016) was based on terrain slope and selected land use classes.

Neither of the approaches described above accommodate situations where issues of accessibility are found in the field, i.e. site inaccessibility is unplanned. Clifford et al. (2014) grappled with this issue and proposed a solution that involves simulated annealing for optimally selecting accessible sites from a region. This method involves multiple criteria such as the KL divergence, ease of access, and geographical coverage, which are all combined into a single criterion. While useful, this approach is computationally demanding to the extent that it would be difficult to apply directly in a field setting. To obviate the need for this, an ordered list of alternative sites 
196 close to each of the primary target sites (should the primary target site prove inaccessible) is

197 produced prior to entering the field.

198 An easier, pragmatic option as demonstrated in the USA by Brungard and Johnanson (2015)

199 would be to calculate a similarity measure to an inaccessible cLHS site within a given buffer 200 zone. In their example, a Gower's dissimilarity index (Gower 1971) was used. With this 201 approach, areas with high similarity to the inaccessible cLHS site could then be identified and 202 the new sample location moved to these areas if needed. Such an approach would allow 203 alternative sites to be determined when in the field, given an appropriate computation device or if 204 areas with high similarity to each location were generated before field sampling begins. This 205 approach is also conveniently provided as a function in the clhs R package by Roudier (2011).

206 In the following, we demonstrate a similar approach to that proposed by Brungard and 207 Johnanson (2015). It differs by generalisation of the metric used to calculate distance between 208 individuals for each of the variables, and it uses a membership function to derive estimates of 209 similarity to a site that has been deemed inaccessible. Flexibility in the selection of distance 210 metric allows alternatives other than the Gower distance to be considered.

211 We demonstrate this approach using a collection of 341 soil samples that cover the Hunter Wine 212 Country Private Irrigation District (HWCPID), a $220 \mathrm{~km}^{2}$ region in the Lower Hunter Valley 213 Region, of New South Wales (NSW), approximately $140 \mathrm{~km}$ north of Sydney, Australia. The 214 description of the sampling and data collection are described in Malone et al. (2014). For this example we used the following ancillary variables derived from a $25 \mathrm{~m}$ digital elevation model: elevation, slope, terrain wetness index, multi-resolution valley bottom flatness, and potential

217 incoming solar radiation. We also used categorical rasterised data from a 1:100 000 geological 218 unit map (Hawley et al. 1995), and a 1:250 000 legacy soil map depicting the surveyed soil units 219 (Kovac and Lawrie 1990) that cover the HWCPID. In this example, each of the 341 sampling 220 locations were assumed to be inaccessible and alternative sites were selected for each.

221 We then compared the KL divergence between the original and alternative sample sites and 222 assess whether the alternative sample sites capture the same environmental information using the 223 following algorithm.

224 Given a inaccessible site location: 
225

226

227

228

229

230

231

232

233

234

235

236

237

238

239

240

241

242

243

244

245

246

247

248

249

250

251

252

1. Create a sampling zone (i.e. buffer) from which an alternative site can be selected and extract all the ancillary data from inside this zone. We used a circled sampling zone of radius $500 \mathrm{~m}$.

2. Calculate the multivariate distance between the inaccessible site and all ancillary data in the sampling zone. If ancillary data is categorical, remove all areas of the sampling zone that do not match the category at the initial sampling location. If ancillary data is continuous calculate a Mahalanobis distance.

3. Transform Mahalanobis distance to a similarity score using a negative sigmoid function:

$$
\text { similarity }=1-\frac{1}{\left.1+e^{\left(1-\times\left(\text { dist }_{i}-\text { dist }_{m e d}\right)\right.}\right)}
$$

Where dist is the Mahalanobis distance of the ancillary data $i$ and the inaccessible site and dist $_{m e d}$ is the median Mahalanobis distance of the available data within the buffer area. We used the median because it is less susceptible to outliers than the mean. The similarity is expressed on a range between 1 and 0 with numbers approaching 1 being highly similar, and is akin to a membership function.

4. Select an alternative site where the similarity is above a given threshold. In our example this threshold was set to 0.975 . Alternatively, one could rank the sites (smallest to highest distance) and select a given number from the top for possible consideration. Whichever the approach, an alternative site may be selected at random from the sites that pass the threshold similarity value, or alternatively the nearest one to the inaccessible site could be selected and so on until it is found that access to the sampling location is possible. In our example we selected the former option.

Repeat for every inaccessible site location

Figure 2 illustrates the steps of this algorithm:

[Insert Figure 2 around here]

Figure 2. Illustrated process of the algorithm for selecting an alternative sampling site when a cLHS site is inaccessible. (A) A sample site (as indicated by a marked point) within the HWCPID area that has been determined to be inaccessible. (B) A circular buffer area is created around the site. If categorical data are being used, those categories that do not match that at the sampling site are excluded. In the given example a circular buffer zone is 
253

254

255

256

257

258

259

260

261

262

263

264

265

266

267

268

269

270

271

272

273

274

275

276

277

278

279

280

281

282

used, but a portion was excluded due to non-matching of the categorical variables. (C) The Mahalanobis distance is estimated between the inaccessible site and all cells in the buffer area. (D) Similarity is estimated using Equation 2 and ranges between 0 and 1 . An alternative sample site is selected if the similarity exceeds 0.975 .

\section{How do I account for existing samples when designing a new survey?}

This is the situation where existing soil samples exist within a sampling domain, and the user wants to derive a sampling configuration that captures environmental variation that the existing samples fails to achieve appropriately. With its current functionality, the clhs R package by Roudier (2011) goes some way to addressing this problem through forming $n 1+n 2$ marginal strata per covariate. Here $n 1$ represents existing site data, and $n 2$ represents marginal strata where new sampling locations can be derived. Essentially one selects a sample size of $n 2$, adds in the existing sample data $(n 1)$ that have previously been collected from the study area, and then runs the clhs algorithm. This appears a useful solution but probably needs to be tested or compared with other approaches such as described below.

Possibly a more explicit approach to dealing with the aforementioned problem, is to first assess the environmental coverage of the existing samples, then look for gaps (where there is no coverage in the data space), and then prioritising new samples to those areas as they occur in the field. This general approach was performed by Stumpf et al. (2016). A more thoroughly described approach is the HELS algorithm and its companion the HISQ algorithm described in Carre et al. (2007). The HELS (Hypercube Evaluation of a Legacy Sample) algorithm is used to check the occupancy of the existing samples in the hypercube of the quantiles of given ancillary data, and to determine whether they occupy the hypercube uniformly or if there is over- or under-observation in partitions of the hypercube. The companion HISQ algorithm is used to preferentially select additional samples in areas where the degree of under-observation is greatest. A limitation of the HELS algorithm is that the full hypercube of all ancillary variables needs to be computed (i.e. if there are $k$ number of variables, $k$-cubes need to be formed). We present an algorithm that is an adaptation and simplification of the combined HELS and HISQ algorithms.

This example uses the same ancillary data and the 341 sample sites from the HWCPID study area described earlier. For this example we want to add an additional 100 samples while 
283 accounting for the prior 341 sample sites. We call this algorithm, adapted HELS (aHELS). A

284 schematic of the aHELS algorithm is shown in Figure 3 and described in the following:

285

286

287

288

289

290

291

292

293

294

295

296

297

298

299

300

301

302

303

304

305

306 0a. Select a sample size of size $s$. In this example $s=100$

0b. Extract the ancillary data values for existing observations $(o)$. In the example $o=341$.

1a. Construct a quantile matrix of the ancillary data. If there are $k$ ancillary variables, the quantile matrix will be of $(s+1) \times k$ dimensions. The rows of the matrix are the quantiles of each ancillary variable.

1b. Calculate the data density of the ancillary data. For each element of the quantile matrix, tally the number of pixels within the bounds of the specified quantile for that matrix element. This number is divided by $r$, where $r$ is the number of pixels of an ancillary variable.

1c. Calculate the data density of ancillary information from the existing legacy samples. This is the same as $1 \mathrm{~b}$ except the number of existing observations are tallied within each quantile (quantile matrix from 1a) and the density is calculated by dividing the tallied number by $o$ instead of $r$.

2. Evaluate the ratio of densities. This is calculated as the point data density divided by the grid density.

3. Rank the density ratios from smallest to largest. Across all elements of the $(s+1) \times k$ matrix of the ratios from step 2, rank them from smallest to largest. The smallest ratios indicate those quantiles that are under-sampled, and should be prioritized for selecting additional sample locations. In this ranking, it is important to save the element row and column indexes.

4a. Begin selection of additional sample locations. Start by initiating a sample of size $s$

4b. While $s>0$ and working down the ranked list of ratios: 
307

308

309

310

311

312

313

314

315

316

317

318

319

320

321

322

323

324

325

326

327

328

329

330

331

332

5. Estimate how many samples $(m)$ are required to make grid density $=$ data density. This is calculated as $o \times$ the grid data density in the same row and column position as the density ratio.

6. Using the same row and column position of the quantile matrix from 1a, select from the grid data all possible locations that meet the criteria of the quantile at that position in the matrix. Select at random from this list of possible locations, $m$ sampling locations.

7. Set $s=s-m$, then go back to $4 \mathrm{~b}$.

[Insert Figure 3 around here]

Figure 3. Schematic of the adapted HELS (aHELS) algorithm. To use this algorithm, one needs their collection of existing site data from the study area, together with a suite of ancillary data rasters. Finally, a specified sample size number $(s)$ needs to be given.

The algorithm will terminate once $s$ sample locations have been selected. A caveat to using the aHELS algorithm is that it is not immediately amenable to optimising sample size as described in the earlier section. Essentially, one needs to select a sample size, then run the algorithm.

Possibly a much more visual way $\square$ yet also complementary to the aHELS algorithm $\square$ to assess relatively adequate and under-sampled areas is to create a map that could show such patterns. We developed an algorithm called COOBS (count of observations) that can achieve this objective. The COOBS algorithm is implemented on a pixel basis and requires a stack of ancillary data pertaining to the environmental variables for a given spatial domain, and the associated legacy data points from the same spatial domain. The legacy points will have been intersected with ancillary data. We demonstrate this COOBS algorithm using the HWCPID example. A schematic of the COOBS algorithm is presented on Figure 4.

1. For each pixel location i: 
333 1a. Calculate the multivariate distance to every other location in the stacked ancillary data. We 334 use the Mahalanobis distance because it preserves the correlation between variables, but other 335 distance or similarity metrics can be considered.

336 1b. Record the maximum distance, which we define as magpd. Note that the minimum distance 337 will always be zero.

338 2. For each pixel location $i$ :

339 2a. Calculate the multivariate distance of the ancillary data between the pixel $i$ and each of the 340 legacy data points. We call this the data distance $(d d)$.

341 2b. Calculate a standardised distance $(s d d)$ for each legacy data point which is simply:

$$
s d d_{i}=1-\frac{d d}{\operatorname{magpd}_{i}}
$$

As the $s d d$ will scale from 0 to 1 , values close to 1 means at least one observation point is similar 344 to the ancillary data for the selected pixel.

3. Set a similarity threshold close to 1 , then count the number of legacy observations that are equal to or better than that criteria. Save this number and pixel location together, then move to the next pixel i.e. go back to Step $2 \mathrm{a}$.

From the above described COOBS algorithm, and depending on how it is implemented, the first step will create a raster of the target area depicting the spatial pattern of magpd. This map is then interrogated pixel-by-pixel in step 2 to estimate the associated COOBS number. Alternatively the two steps of the COOBS algorithm could be implemented as a single workflow whereby the maps of magpd and COOBS are derived at near the same time as each other, pixel-by-pixel.

[Insert Figure 4 around here]

Figure 4. Schematic of the COOBS algorithm. To use this algorithm, one needs their collection of existing site data from the study area, together with a suite of ancillary data rasters. 
360

361

362

363

364

365

366

367

368

369

370

371

372

373

374

375

376

377

378

379

380

381

382

383

384

385

386

387

\section{Results and Discussion}

How many samples should I collect?

The resulting output is shown in Figure 5A which is a plot of the mean KL divergence with increasing sample size and is a classical exponential decay with increasing sample size. We do not show the standard deviation bars on this plot as they are very small with increasing sample size and are not visible on the plot. However, relatively larger standard deviations were observed at smaller sample sizes which decreased with increasing sample sizes. We also calculated the KL divergence for the 238 samples already collected from the field of interest. This was found to be 0.039 , and as can be seen on Figure 5A as the '+' symbol. This mark is above the fitted line for the same sample size using cLHS. This indicates that (for this example), cLHS is superior to grid sampling for capturing the variation in ancillary data for the same sample size.

To estimate an optimal sample size, we derived a cumulative density function for the fitted line on Figure 5A, and identified the sample size required to capture $95 \%$ of the cumulative probability (Figure 5B). The optimal sample size was 110. The KL divergence for this sample size was 0.033 , slightly lower (better) than that achieved with the actual 238 grid samples.

In a practical setting, the 110 samples would be used as the minimum recommended sample size. However, one caveat is that other metrics of comparison are likely to result in differing optimal sample sizes because the underlying calculation is different. In situations where multiple comparison metrics are used, it might be pragmatic to assess what the minimum and maximum optimal sample sizes are, then select a recommended sample size in the middle. Though likely not optimal, this approach would be an improvement on the arbitrary selection of sample size that currently occurs in most studies.

[Insert Figure 5 around here]

Figure 5. A) KL divergence between ancillary data of a sample and ancillary data of the population as a function of sample size. The fitted line is a negative exponential decay function fit to the KL divergence with the relationship $\mathrm{y}=\mathrm{b} \_1 \cdot\left[\exp \rrbracket^{\wedge}((-\mathrm{kx}))+\mathrm{b} \_0\right.$ where $\mathrm{x}$ and $\mathrm{y}$ were sample size and KL divergence respectively. The fitted parameters of $\mathrm{b} \_0, \mathrm{~b} \_1$, and $\mathrm{k}$ were $0.275,1.185$, and 0.028 respectively. The '+' symbol is the KL divergence for a grid sample 
388

389

390

391

392

393

394

395

396

397

398

399

400

401

402

403

404

405

406

407

408

409

410

411

412

413

414

415

of 239. B) Cumulative density function of the $1-\mathrm{KL}$ divergence (from 5A) as a function of sample size. The point of intersection of the two straight lines is the optimal sample size as this is the point where the cdf breaches $95 \%$.

Where else can I sample when a cLHS location cannot be visited because of difficult terrain, locked gate, safety reasons, etc.?

Table 1 presents the KL divergences for each ancillary variable for both the original sample configuration and the alternative sample configuration for each of the 341 sites. Overall, the mean KL divergence is equal for both sampling configurations which is a desired outcome of the algorithm. With this relatively simple procedure or adopting the similar approach described in Brungard and Johnanson (2015) it becomes possible to derive alternative sample locations within the field at the time of sampling. This delivers to the field technician an objective way to select an alternative sample site (or possibly even a general area) during the survey campaign.

\section{[Insert table 1 around here]}

Table 1. KL divergence of the original sample configuration and the relocated sample configuration (341 sites) for each of the ancillary data and an overall mean.

\section{How do I account for existing samples when designing a new survey?}

Figure 6A shows a map with the original 341 sites and the additional 100 sites selected using the aHELS algorithm. The COOBS algorithm created the map on Figure 6B. A caveat to the COOBS algorithm though is that it is computationally heavy. Some of this burden can easily be diffused via compute parallelisation however. Alternatively, and in order to take advantage of spatial correlation, it could be possible to take a representative sample from the provided ancillary data of a specified size, calculate magpd for each of these locations, then interpolate this target variable across the whole area. Future investigations would need to assess the viability and efficiency of this approach.

$<$ Figure 6 around here $>$ 
416 Figure 6. A) HWCPID existing 341 sites (crosses) and additional 100 sites (dots) selected using the adapted HELS

417 algorithm. B) Existing sample data coverage using the 341 HWCPID sites based on using the COOBS algorithm.

418 Values indicate the number of existing soil samples that are above a similarity threshold for each pixel. Lower

419 values indicate areas with fewer similar observations and which likely require additional sampling.

420

421 Overall, both the aHELS and $C O O B S$ algorithms allow one to understand which areas in a 422 spatial domain are adequately and under-sampled. The aHELS algorithm is explicit in selecting 423 sites preferentially to locations in the environment that are not captured by the existing site data.

424 Relatedly, the COOBS algorithm provides a visualisation of general areas where under sampling 425 is prevalent. As an aside, it may be possible to use the COOBS derived map to design an 426 additional survey by constraining the cLHS algorithm to areas where the COOBS value is below some specified threshold. Nevertheless, both aHELS and COOBS are somewhat comparable in their objectives and ultimately generate similar outcomes as shown in Table 2 where the

429 proportion of sample sites selected using the aHELS is much higher in the areas where the 430 COOBS algorithm determined relatively low existing data coverage. For example $87 \%$ of additional sites appear to be allocated in areas where the existing data coverage is $<=10$ sites.

432

433

434

435

436

437

438

439

440

441

442

443

444

445

$<$ Table 2 around here $>$

Table 2. Proportions of original and additional sampling sites in the HWCPID selected using the adapted HELS algorithm that occur within groupings of the sample data coverage determined using the COOBS algorithm. Note that COOBS means at the pixel level, the count of observations estimated to be similar in terms of the given ancillary data.

\section{Conclusions}

This technical note provides some solutions to common questions that arise when the cLHS algorithm is used for designing a soil or any other environmental survey. We have collated solutions that others have come up with to deal with such questions, and also presented new and/or modified solutions too. We have presented a solution to optimise a sample size number and to take into consideration existing soil samples. Importantly we have introduced a relatively simple approach to relocate a site for soil sampling in situations of inaccessibility without deteriorating the original cLHS site configuration. R scripts with associated data examples for 
446

447

448

449

450

451

452

453

454

455

456

457

458

459

460

461

462

463

464

465

466

467

468

469

470

471

472

473

474

475

each of these solutions is shared in a data repository at:

https://bitbucket.org/brendo1001/clhc sampling

\section{References}

Brungard, C., Johnanson, J., 2015. The gate's locked! I can't get to the exact sampling spot ... can I sample nearby? Pedometron: Newsletter of the Pedometrics Commission of the IUSS. Pedometron (37): Newsletter of the Pedometrics Commission of the IUSS. URL: http://www.pedometrics.org/Pedometron/Pedometron37.pdf, Sydney, Australia.

Carré, F., McBratney, A.B., Minasny, B., 2007. Estimation and potential improvement of the quality of legacy soil samples for digital soil mapping. Geoderma 141(1), 1-14.

Chu, H.-J., Chen, R.-A., Tseng, Y.-H., Wang, C.-K., 2014. Identifying LiDAR sample uncertainty on terrain features from DEM simulation. Geomorphology 204, 325-333.

Clifford, D., Payne, J.E., Pringle, M.J., Searle, R., Butler, N., 2014. Pragmatic soil survey design using flexible Latin hypercube sampling. Computers \& Geosciences 67, 62-68.

de Gruijter J, Brus D, Bierkens M, Knotters M. 2006. Sampling for Natural Resource Monitoring: Statistics and Methodology of Sampling and Data Analysis. Berlin: Springer-Verlag. 343 pp.

Gower, J.C., 1971. A General Coefficient of Similarity and Some of Its Properties. Biometrics 27(4), 857-871.

Hawley, S.P., Glen, R.A., Baker, C.J., 1995. Newcastle Coalfield Regional Geology 1:100 000, 1st Edition. Geological Survey of New South Wales, Sydney, Australia.

Jeong, G., Choi, K., Spohn, M., Park, S.J., Huwe, B., Ließ, M., 2017. Environmental drivers of spatial patterns of topsoil nitrogen and phosphorus under monsoon conditions in a complex terrain of South Korea. PLOS ONE 12(8), e0183205.

Kidd, D., Malone, B., McBratney, A., Minasny, B., Webb, M., 2015. Operational sampling challenges to digital soil mapping in Tasmania, Australia. Geoderma Regional 4, 1-10.

Kopačková, V., Ben-Dor, E., Carmon, N., Notesco, G., 2017. Modelling Diverse Soil Attributes with Visible to Longwave Infrared Spectroscopy Using PLSR Employed by an Automatic Modelling Engine. Remote Sensing 9(2), 134. 
476 Kovac, M., Lawrie, J.M., 1990. Soil Landscapes of the Singleton 1:250 000 Sheet. Soil

477 Conservation Service of NSW, Sydney, Australia.

478 Krzanowski, W.J., 1979. Between-Groups Comparison of Principal Components. Journal of the $479 \quad$ American Statistical Association 74(367), 703-707.

480 Kullback, S., Leibler, R.A., 1951. On Information and Sufficiency, 79-86.

481 Malone, B.P., Hughes, P., McBratney, A.B., Minasny, B., 2014. A model for the identification of 482 terrons in the Lower Hunter Valley, Australia. Geoderma Regional 1, 31-47.

483 McBratney, A.B., Mendonça Santos, M.L., Minasny, B., 2003. On digital soil mapping. 484 Geoderma 117(1), 3-52.

McKay, M.D., Beckman, R.J., Conover, W.J., 1979. A Comparison of Three Methods for Selecting Values of Input Variables in the Analysis of Output from a Computer Code. Technometrics 21(2), 239-245.

Minasny, B., McBratney, A.B., 2006. A conditioned Latin hypercube method for sampling in the presence of ancillary information. Computers \& Geosciences 32(9), 1378-1388.

Mulder, V.L., de Bruin, S., Schaepman, M.E., 2013. Representing major soil variability at regional scale by constrained Latin hypercube sampling of remote sensing data. International Journal of Applied Earth Observation and Geoinformation 21, 301-310.

Ramirez-Lopez, L., Schmidt, K., Behrens, T., van Wesemael, B., Demattê, J.A.M., Scholten, T., 2014. Sampling optimal calibration sets in soil infrared spectroscopy. Geoderma 226227, 140-150.

Roudier, P., 2011. clhs: a R package for conditioned Latin hypercube sampling.

Roudier, P., Beaudette, D., Hewitt, A., 2012. A conditioned Latin hypercube sampling algorithm incorporating operational constraints. In: B. Minasny, B. Malone, A.B. McBratney (Eds.), Digital Soil Assessments and Beyond: Proceedings of the 5th Global Workshop on Digital Soil Mapping. CRC Press, The Netherlands, pp. 227-231.

502

Scarlett, K., Daniel, R., Shuttleworth, L.A., Roy, B., Bishop, T.F.A., Guest, D.I., 2015. Phytophthora in the Gondwana Rainforests of Australia World Heritage Area. Australasian Plant Pathology 44(3), 335-348.

Scarpone, C., Schmidt, M.G., Bulmer, C.E., Knudby, A., 2016. Modelling soil thickness in the critical zone for Southern British Columbia. Geoderma 282, 59-69. 
506 Shannon CE, Weaver W. 1949. Mathematical Theory of Communication. pp 117. Urbana: Univ. 507 of Illinois Press.

508 Stumpf, F., Schmidt, K., Behrens, T., Schönbrodt-Stitt, S., Buzzo, G., Dumperth, C., Wadoux, 509 A., Xiang, W., Scholten, T., 2016. Incorporating limited field operability and legacy soil 510 samples in a hypercube sampling design for digital soil mapping. Journal of Plant $511 \quad$ Nutrition and Soil Science 179(4), 499-509.

512 Sun, X.-L., Wang, H.-L., Zhao, Y.-G., Zhang, C., Zhang, G.-L., 2017. Digital soil mapping 513 based on wavelet decomposed components of environmental covariates. Geoderma 303, $514 \quad 118-132$.

515 Thomas, M., Clifford, D., Bartley, R., Philip, S., Brough, D., Gregory, L., Willis, R., Glover, M., 516 2015. Putting regional digital soil mapping into practice in Tropical Northern Australia. 517 Geoderma 241-242, 145-157.

518 Thomas, M., Odgers, N., Ringrose-Voase, A., Grealish, G., Glover, M., Dowling, T., 2012. Soil 519 survey design for management-scale digital soil mapping in a mountainous southern 520 Philippine catchment. In: B. Minasny, B.P. Malone, A.B. McBratney (Eds.), Digital Soil Assessments and Beyond: Proceedings of the 5th Global Workshop on Digital Soil

523 Webster R, Lark M. 2013. Field Sampling for Environmental Science and Management. pp 192. New York, NY: Routledge 


\section{Figure 1}

Schematic of sample number size optimisation algorithm.

Figure 1. Schematic of sample number size optimisation algorithm. 


\section{Identify target area and collate ancillary data.}

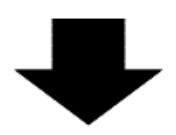

\section{Conditioned Latin hypercube sampling.}

- Repeat CLHC sampling $x$ number of times, with sequentially increasing sample size. e.g. samples sizes for 10 to 500 in increments of 10

- For each sample size, record KL divergence [population ancillary data vs. sample ancillary data]

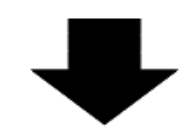

\section{Determine optimal sample size.}

- Make an xy-plot of number of samples vs. KLdivergence.

- Fit exponential decay function to plotted data.

- Derive cumulative density function (cdf) of the fitted exponential of $1-K L$ divergence.

- Optimal sample size is the number of the cdf breaches the 95\% probability level. 


\section{Figure 2}

\section{Selection of alternate sites}

Figure 2. Illustrated process of the algorithm for selecting an alternative sampling site when a cLHS site is inaccessible. (A) A sample site (as indicated by a marked point) within the HWCPID area that has been determined to be inaccessible. (B) A circular buffer area is created around the site. If categorical data are being used, those categories that do not match that at the sampling site are excluded. In the given example a circular buffer zone is used, but a portion was excluded due to non-matching of the categorical variables. (C) The Mahalanobis distance is estimated between the inaccessible site and all cells in the buffer area. (D) Similarity is estimated using Equation 2 and ranges between 0 and 1 . An alternative sample site is selected if the similarity exceeds 0.975 . 
(A)

(B)

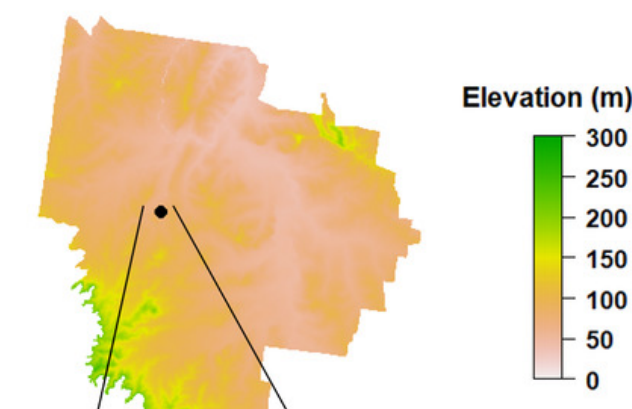

(C)

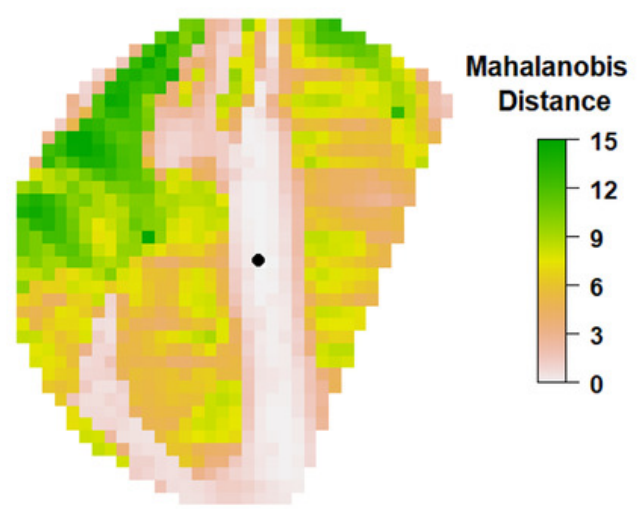

(D)

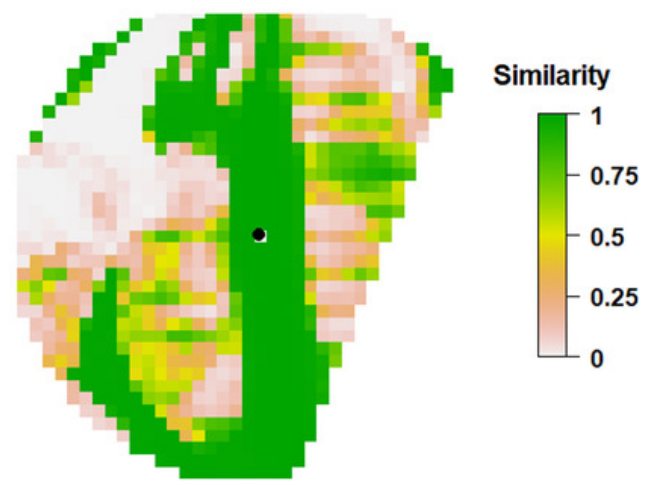




\section{Figure 3}

\section{Adapted HELS algorithm}

Figure 3. Schematic of the adapted HELS (aHELS) algorithm. To use this algorithm, one needs their collection of existing site data from the study area, together with a suite of ancillary data rasters. Finally, a specified sample size number (s) needs to be given.

*Note: Auto Gamma Correction was used for the image. This only affects the reviewing manuscript. See original source image if needed for review. 
Figure 4

\section{COOBS Algorithm}

Figure 4. Schematic of the COOBS algorithm. To use this algorithm, one needs their collection of existing site data from the study area, together with a suite of ancillary data rasters.

For each pixel:

1. Calculate multi-variate distance (using ancillary data) to all other pixels.

2. Record maximum distance (magpd)

\section{For each pixel:}

1. For all sites data points, estimate multivariate distance to selected pixel.

2. Standardise distance

3. Count number of sites that occur within critical distance of similarity to selected pixel.

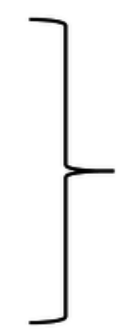

Step 1. Pixel-topixel comparisons

Step 2. Pixel-todata point comparisons 


\section{Figure 5}

$\mathrm{KL}$ divergence as function of sample size

Figure 5. A) KL divergence between ancillary data of a sample and ancillary data of the population as a function of sample size. The fitted line is a negative exponential decay function fit to the $K L$ divergence with the relationship $y=b_{-} 1 \cdot \square$ exp ${ }^{\wedge}((-k x))+b_{-} 0$ where $x$ and $y$ were sample size and $K L$ divergence respectively. The fitted parameters of $b_{-} 0, b_{-} 1$, and $k$ were $0.275,1.185$, and 0.028 respectively. The ' + ' symbol is the $K L$ divergence for a grid sample of 239. B) Cumulative density function of the $1-K L$ divergence (from $5 A$ ) as a function of sample size. The point of intersection of the two straight lines is the optimal sample size as this is the point where the cdf breaches $95 \%$. 

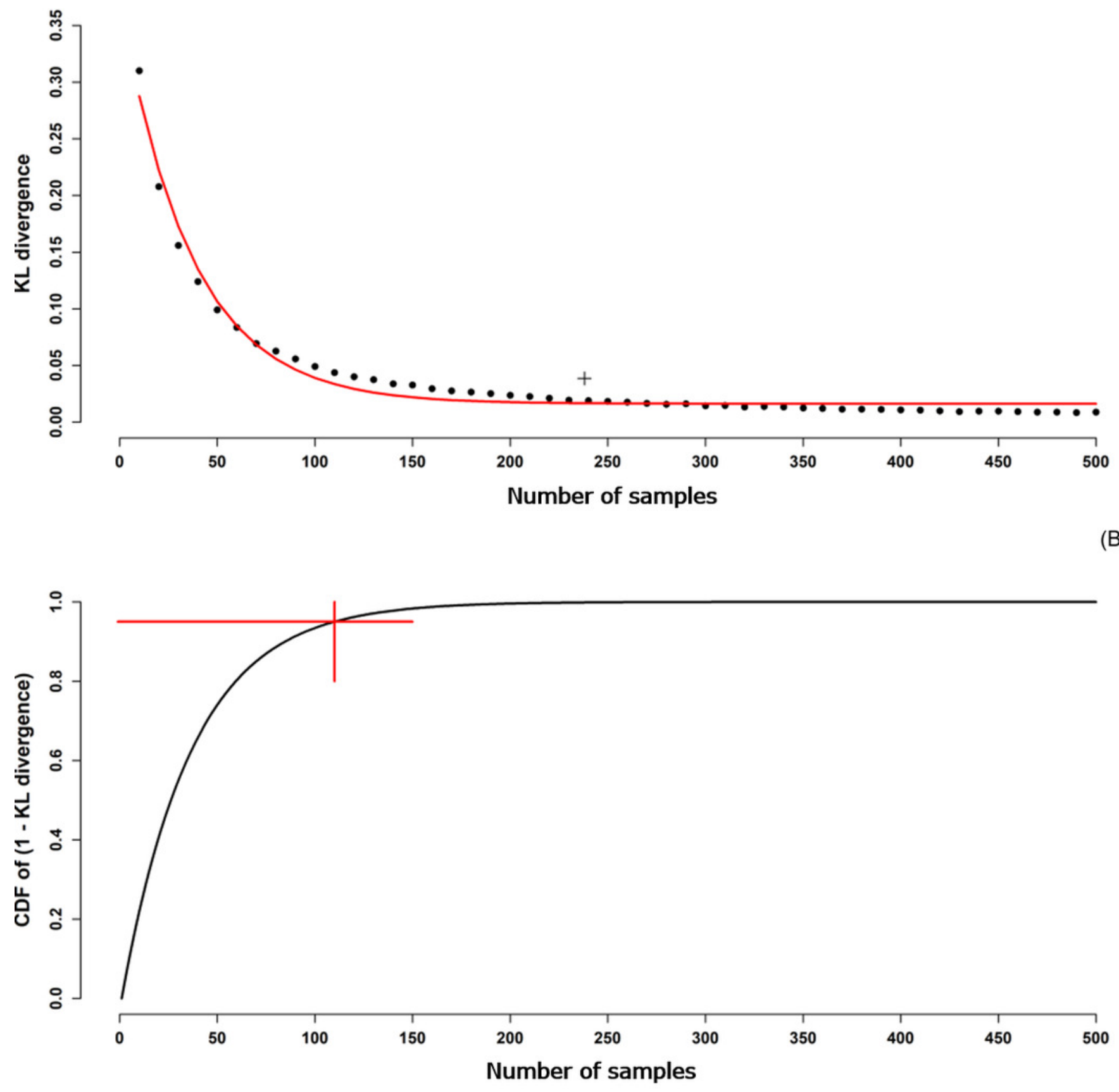


\section{Figure 6}

Comparative outputs of aHELS and COOBS algorithms

Figure 6. A) HWCPID existing 341 sites (crosses) and additional 100 sites (dots) selected using the adapted HELS algorithm. B) Existing sample data coverage using the 341 HWCPID sites based on using the COOBS algorithm. Values indicate the number of existing soil samples that are above a similarity threshold for each pixel. Lower values indicate areas with fewer similar observations and which likely require additional sampling.

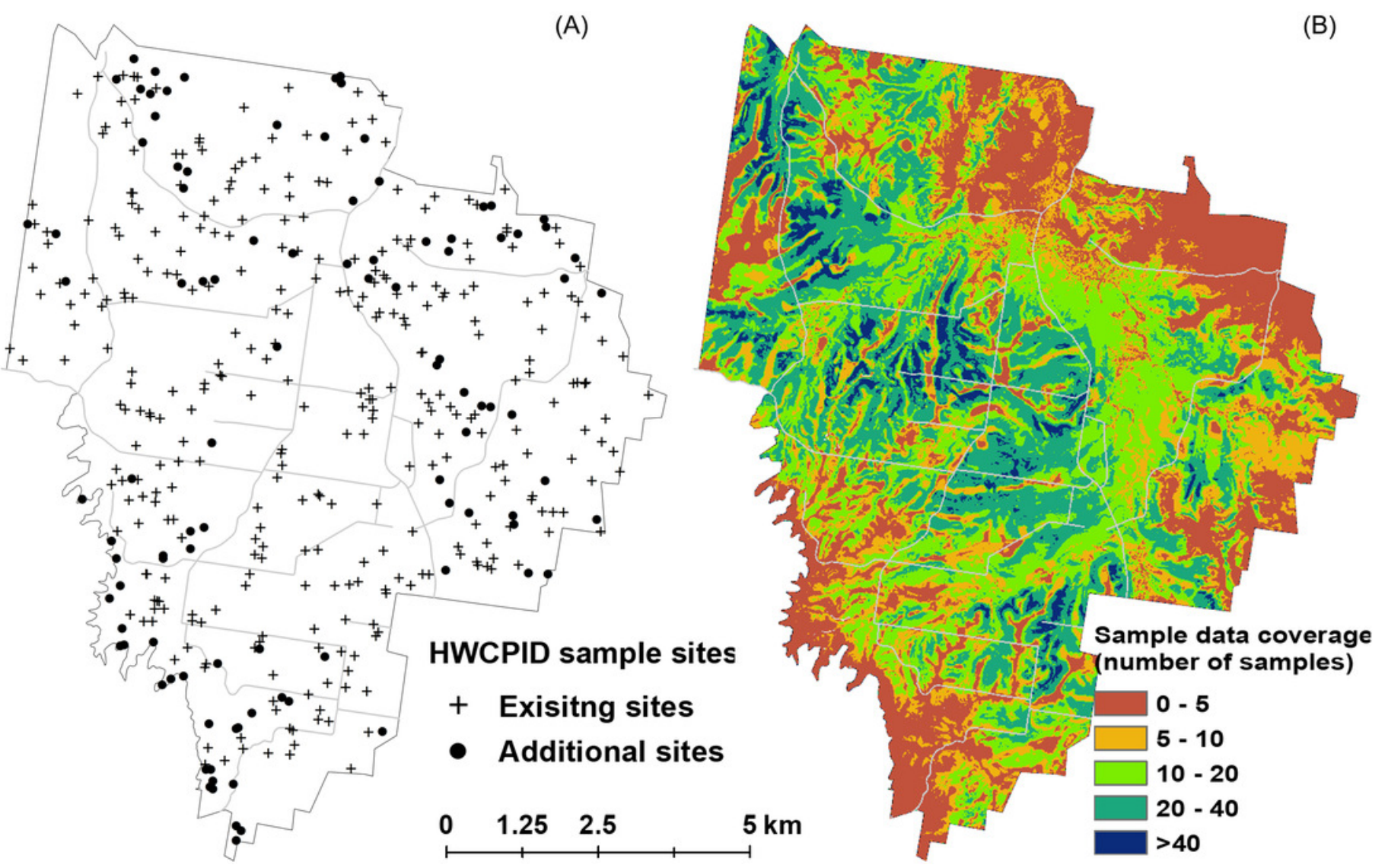




\section{Table $\mathbf{1}$ (on next page)}

KL-divergence comparison between original sample and optimised sample.

$\mathrm{KL}$-divergence of the original sample configuration and the relocated sample configuration (341 sites) for each of the ancillary data and an overall mean. 


\begin{tabular}{lcc}
\hline Ancillary variable & KL-divergence (original) & KL-divergence (relocated) \\
\hline Terrain Wetness Index & 0.021 & 0.019 \\
(unitless) & & \\
Slope (degree) & 0.038 & 0.041 \\
Multi-resolution Valley & 0.009 & 0.019 \\
Bottom Flatness (unitless) & & \\
Potential Incoming Solar & 0.063 & 0.052 \\
Radiation (kWh/m2) & & \\
Elevation (meters) & 0.034 & 0.033 \\
\hline mean & $\mathbf{0 . 0 3 3}$ & $\mathbf{0 . 0 3 3}$
\end{tabular}

1 


\section{Table 2 (on next page)}

Similarity between adapted HELS and coobs algorithm outputs

Proportions of original and additional sampling sites in the HWCPID selected using the adapted HELS algorithm that occur within groupings of the sample data coverage determined using the coobs algorithm. Notes that coobs means at the pixel level, the count of observations estimated to be similar in terms of the given ancillary data. 


\begin{tabular}{ccc}
\hline $\begin{array}{l}\text { Legacy sample data } \\
\text { coverage (derived from } \\
\text { coobs algorithm }\end{array}$ & $\begin{array}{l}\text { Proportions of allocated } \\
\text { adapted HELS sites (100) }\end{array}$ & $\begin{array}{l}\text { Proportions of existing } \\
\text { sites (341) }\end{array}$ \\
\hline $0-5$ & 0.66 & 0.26 \\
$5-10$ & 0.21 & 0.17 \\
$10-20$ & 0.12 & 0.30 \\
$20-40$ & 0.01 & 0.23 \\
$>40$ & 0.00 & 0.03 \\
\hline
\end{tabular}

1 